\title{
ON THE FIRST COHOMOLOGY GROUP OF DISCRETE GROUPS WITH PROPERTY (T)
}

\author{
S. P. WANG
}

\begin{abstract}
Let $G$ be a separable locally compact group with property $(T)$, i.e., the class of one dimensional trivial representations is an isolated point in the dual space $\hat{G}$ of $G$. Let $\pi: G \rightarrow O_{n}$ be a continuous representation of $G$ into the orthogonal group. In this note, we show that $H^{1}(G, \pi)=0$.
\end{abstract}

Let $G$ be a separable locally compact group. Let $\hat{G}$ be the set of all equivalence classes of separable irreducible unitary representations of $G$. We give $\hat{G}$ the inner-hull-kernel topology. $\hat{G}$ is called the dual space of $G$. Following [4], $G$ is said to have property $(\mathrm{T})$ if the equivalence class $I$ of the one dimensional trivial representation of $G$ is an isolated point in $\hat{G}$. Let $\pi: G \rightarrow O_{n}$ be a continuous representation of $G$ into the group of all $n$ by $n$ orthogonal matrices. Let us write $C^{1}(G, \pi)$ and $B^{1}(G, \pi)$ for the groups of continuous 1-cocycles and 1-coboundaries respectively. Let $H^{1}(G, \pi)=C^{1}(G, \pi) / B^{1}(G, \pi)$. In this note, we are going to prove that $H^{1}(G, \pi)=0$ if $G$ is a separable locally compact group with property $(\mathrm{T})$. Applying to discrete subgroups of $p$-adic groups or real Lie groups, our result generalizes slightly some results in [4] and [3, Theorem 6.5]. Our argument still follows the spirit of [4]. Before we give the proof of the main result, we shall first establish some lemmas needed later.

LEMMA 1. Let $H$ be a locally compact group and $\pi: H \rightarrow O_{n}$ a representation. Let $\psi: H \rightarrow R^{n}$ be a 1-cocycle. If $\varphi(H)$ is bounded in $R^{n}$, then $\varphi$ is a coboundary.

Proof. Let $K$ be the closure of $\pi(H)$ in $O_{n}$ and $K \cdot R^{n}$ the semidirect product of $K$ and $R^{n}$ where $R^{n}$ is the normal subgroup and $K$ acts on $R^{n}$ in the natural manner. Consider then the $\operatorname{map} f: H \rightarrow K \cdot R^{n}$ defined by $h \rightarrow \pi(h) \psi(h)(h \in H)$. Since $\psi$ is a 1-cocycle, $f$ clearly is a homomorphism. $\phi(H)$ is bounded by assumption. It yields that $\mathrm{Cl}(f(H))$ is compact.

Received by the editors January 30, 1973.

AMS (MOS) subject classifications (1970). Primary $22 \mathrm{E} 40$.

Key words and phrases. Locally compact groups, groups with property (T), affine semisimple algebraic groups.

1 Partially supported by NSF Grant GP-29466X. 
By the conjugacy theorem, there is $m$ in $R^{n}$ such that $m \mathrm{Cl}(f(H)) m^{-1} \subset K$. In particular, we have

$$
m \pi(h) \varphi(h) m^{-1}=\pi(h)\left(m^{h} \varphi(h) m^{-1}\right) \in K,
$$

where $m^{h}=\pi(h)^{-1} m \pi(h)$ and $h \in H$. Hence $m^{h} \varphi(h) m^{-1}=1$ and in additive notation, we get $\varphi(h)=m-m^{h}, h \in H$. Therefore $\varphi$ is a coboundary.

LEMMA 2. Let $G$ be a separable locally compact group with property (T). Then there exist a positive number $\varepsilon$ and a compact subset $K$ of $G$ with the following condition: If $\pi$ is a separable unitary representation of $G$ on a Hilbert space $H(\pi)$ and $x \in H(\pi)$ such that $\|x\|=1$ and $|(g x, x)-1|<\varepsilon$ for all $g \in K$, then $\pi \geqq I$.

Proof. Suppose the assertion to be false. Let $K_{n}$ be an increasing sequence of compact subsets of $G$ such that $\bigcup_{n} K_{n}=G$. Then there are separable unitary representations of $G, \pi_{1}, \pi_{2}, \cdots$ for which there exist $x_{n} \in H\left(\pi_{n}\right)$ with $\left\|x_{n}\right\|=1,\left|\left(g x_{n}, x_{n}\right)-1\right|<1 / n$ for $g \in K_{n}$ and $\pi_{n} \geq I$ $(n=1,2, \cdots)$. Then consider the representation $\pi=\bigoplus_{i=1}^{\infty} \pi_{i}$. Due to our construction, $I$ is contained in the closure of $\{\pi\} .^{2}$ However $G$ has property (T), by [4], [6], $I \leqq \pi$ which implies $I \leqq \pi_{n}$ for some $n$. Obviously this is a contradiction.

Lemma 3. Let $G, \varepsilon, K$ be described as in Lemma 2. Let $\pi$ be a separable unitary representation of $G$ in the Hilbert space $H(\pi)$. If $x \in H(\pi)$ with $\|x\|=1$ and $|(g x, x)-1|<\varepsilon^{2}$ for $g \in K$, then $\|g x-x\|<2 \sqrt{ } \varepsilon$ for all $g \in G$.

Proof. Let us write $H_{1}$ for the set $\{y \in H(\pi): g y=y$ for all $g$ in $G\}$ and $H_{2}=H_{1}^{\perp}$. Clearly $H_{2}$ is invariant under $G$, and $H(\pi)=H_{1} \oplus H_{2}$. It is also easy to see that $\left.\pi\right|_{H_{2}} I$. Let us write $x=x_{1}+x_{2}$ with $x_{1} \in H_{1}$ and $x_{2} \in H_{2}$. Since $g x=x_{1}+g x_{2}$ and $\|x\|=1$,

$$
i(g x, x)-1|=|\left(g x_{2}, x_{2}\right)-\left(x_{2}, x_{2}\right) \mid<\varepsilon^{2} \quad \text { for } g \in K .
$$

Since $\left.\pi\right|_{H_{2}} I$ and by Lemma 2 , we must have $\left\|x_{2}\right\|^{2}<\varepsilon$. From this, $\|g x-x\|=\left\|g x_{2}-x_{2}\right\|<2 \sqrt{ } \varepsilon$, for all $g \in G$.

LEMMA 4. Let $G$ be a separable locally compact group with property (T), $\pi: G \rightarrow O_{n}$ a continuous homomorphism and $\varphi: G \rightarrow R^{n} a$ continuous 1-cocycle. Then $\varphi(G)$ is bounded in $R^{n}$.

ProOF. Let $\varphi: G \rightarrow R^{n}$ be a continuous 1-cocycle. We define $\alpha_{\lambda}: G \rightarrow$ $G \cdot R^{n}$ (semidirect product) by $g \rightarrow g(\lambda \varphi(g))(g \in G)$. Clearly the maps

\footnotetext{
${ }^{2}$ Let $\tilde{G}$ be the set of all equivalence classes of separable unitary representations of $G$. We give $\tilde{G}$ the inner hull-kernel topology.
} 
$\alpha_{\lambda}(0 \leqq \lambda \leqq 1)$ are continuous homomorphisms and, as $\lambda \rightarrow 0, \alpha_{\lambda} \rightarrow$ the inclusion map $i_{G}$ of $G$ in $G \cdot R^{n}$. Now consider the space $L^{2}\left(G \backslash G \cdot R^{n}\right)=$ $L^{2}\left(R^{n}\right)$. Through right translations, $G \cdot R^{n}$ acts unitarily on $L^{2}\left(G \mid G \cdot R^{n}\right)$. Let $B$ be a unit volume ball in $R^{n}$ with center at 0 , and $x_{B}$ the characteristic function on $B$. Since $B$ is invariant under $O_{n}, g x_{B}=x_{B}$ for all $g \in G$. Now let $\varepsilon<\frac{1}{2}$, and $G, K$ be described as in Lemma 2. Since $\alpha_{\lambda} \rightarrow i_{G}$ as $\lambda \rightarrow 0$, there is $\delta>0$ such that

$$
\left|\left(\alpha_{\lambda}(g) x_{B}, x_{B}\right)-1\right|<\varepsilon^{2}, \quad g \in K,
$$

and $0 \leqq \lambda \leqq \delta$. By Lemma $3,\left\|\alpha_{\lambda}(g) x_{B}-x_{B}\right\|<2 \sqrt{ } \varepsilon$ for all $g \in G$ and $0 \leqq$ $\lambda \leqq \delta$. By an easy computation, $\alpha_{\lambda}(g) x_{B}=x_{B^{1}}$, the characteristic function on $B^{1}$ where $B^{1}$ is the translation of $B$ by a vector $-\lambda \varphi(g)^{g^{-1}}$. Note $-\lambda \varphi(g)^{g^{-1}}=-\lambda \pi\left(g^{-1}\right)(\varphi(g))$ has norm $\|\lambda \varphi(g)\|$. If $\|\lambda \varphi(g)\| \geqq 1, B \cap B^{1}=\varnothing$, hence $\left\|\alpha_{\lambda}(g) x_{B}-x_{B}\right\|=\sqrt{ } 2>2 \sqrt{ } \varepsilon$. However

$$
\left\|\alpha_{\lambda}(g) x_{B}-x_{B}\right\|<2 \sqrt{ } \varepsilon \text { for all } g \in G
$$

and $0 \leqq \lambda \leqq \delta$. It follows that $\|\delta \varphi(g)\|<1$ for all $g \in G$. Hence $\varphi(G)$ is bounded in $R^{n}$.

As a consequence of Lemmas 1 and 4, we now have our main result.

THEOREM A. Let $G$ be a separable locally compact group with property (T) and $\pi: G \rightarrow O_{n}$ a continuous representation. Then $H^{1}(G, \pi)=0$.

As an application of Theorem $\mathrm{A}$, we have the following vanishing cohomology theorem of certain discrete subgroups.

THEOREM B. Let $k$ be a nondiscrete locally compact field of $\operatorname{ch}(k)=0$, $G$ an affine semisimple algebraic group defined over $k$ and $\Gamma$ a discrete subgroup of $G(k)$. If the $k$-rank of each $k$-factor of $G \geqq 2$ and $G(k) / \Gamma$ has $a$ finite Haar measure, then $H^{1}(\Gamma, \pi)=0$ for every finite dimensional unitary representation $\pi$ of $\Gamma$.

Proof. By [4], $\Gamma$ has property ( $\mathrm{T})$.

Theorem B generalizes slightly Theorem 6.5 in [3]. However our method cannot be carried out in higher dimensional cohomology groups. In the following, we present a weak rigidity theorem for discrete groups with property $(\mathrm{T})$.

THEOREM C. Let $\Gamma$ be a finitely generated discrete group with property (T), $G$ a compact Lie group, and $\varphi_{\lambda}: \Gamma \rightarrow G(0 \leqq \lambda \leqq 1)$ a continuous curve of homomorphisms. Then for each $\lambda$, there is $g_{\lambda} \in G$ such that $\varphi_{\lambda}(\gamma)=$ $g_{\lambda} \varphi_{0}(\gamma) g_{\lambda}^{-1}$ for all $\gamma \in \Gamma$.

PROOF. Let $n$ be a positive integer. From [7], we know that there are only finitely many classes of irreducible unitary representations of $\Gamma$ 
with dimension $\leqq n$. Let $N_{\lambda}=\operatorname{kernel}\left(\varphi_{\lambda}\right)$. Then the set of kernels $\left\{N_{\lambda}: 0 \leqq\right.$ $\lambda \leqq 1\}$ is finite. Let us denote this finite set by $\left\{M_{1}, \cdots, M_{l}\right\}$. Let $I_{i}=$ $\left\{\lambda: N_{\lambda}=M_{i}\right\}$. We want to show that $I_{i},(i=1, \cdots, l)$ are closed subsets of $[0,1]$. Let us write $\Gamma_{i}$ for $\Gamma / M_{i}$. Clearly if $\lambda \in \mathrm{Cl}\left(I_{i}\right), N_{\lambda} \supset M_{i}$. Hence for each $\lambda \in \mathrm{Cl}\left(I_{i}\right)$, we set $\bar{\varphi}_{\lambda}$ for the homomorphism of $\Gamma / M_{i}$ induced by $\varphi_{\lambda}$. Let $\mathscr{R}\left(\Gamma_{i}, G\right)$ be the space of all representations of $\Gamma_{i}$ in $G$. We equip $\mathscr{R}\left(\Gamma_{i}, G\right)$ with the compact open topology. $G$ acts continuously on $\mathscr{R}\left(\Gamma_{i}, G\right)$ by $(g \circ \varphi)(\gamma)=g \varphi(\gamma) g^{-1}, \varphi \in \mathscr{R}\left(\Gamma_{i}, G\right), \gamma \in \Gamma_{i}$ and $g \in G$. By Theorem $\mathrm{A}$ and $[\mathbf{8}], G \circ \bar{\varphi}_{\lambda},\left(\lambda \in I_{i}\right)$ are open subsets of $\mathscr{R}\left(\Gamma_{i}, G\right)$. Since $G$ is compact, $G \circ \bar{\varphi}_{\lambda},\left(\lambda \in I_{i}\right)$ are compact-open in $\mathscr{R}\left(\Gamma_{i}, G\right)$. On the other hand $\mathscr{R}\left(\Gamma_{i}, G\right)$ can be viewed as an $R$-algebraic variety. It follows that $\mathscr{R}\left(\Gamma_{i}, G\right)$ has only finitely many arcwise connected components [9]. Hence

$$
\bigcup_{\lambda \in I_{i}} G \circ \bar{\varphi}_{\lambda}=\bigcup_{j=1}^{m} G \circ \bar{\varphi}_{\lambda_{j}}
$$

for some finitely many elements $\lambda_{1}, \cdots, \lambda_{m}$ in $I_{i}$, and consequently $\bigcup_{\lambda \in I_{i}} G \circ \bar{\varphi}_{\lambda}$ is compact-open in $\mathscr{R}\left(\Gamma_{i}, G\right)$. Therefore $\mathrm{Cl}\left(I_{i}\right)=I_{i}(i=$ $1, \cdots, l)$ and $l$ has to be 1 . Again by Theorem A and [8] $G \circ \bar{\varphi}_{0}$ is compact-open in $\mathscr{R}\left(\Gamma_{1}, G\right)$. Since $\left\{\bar{\varphi}_{\lambda}: 0 \leqq \lambda \leqq 1\right\}$ is connected, one concludes readily that $\bar{\varphi}_{\lambda} \in G \circ \bar{\varphi}_{0}$ for all $\lambda$. Therefore $\varphi_{\lambda} \in G \circ \varphi_{0}$ for all $\lambda$.

\section{REFERENCES}

1. A. Borel, Density properties of certain subgroups of semi-simple groups without compact components, Ann. of Math. (2) 72 (1960), 179-188. MR 23 \#A964.

2. J. D. Dixmier, Points isolés dans le dual d'un groupe localement compact, Bull. Sci. Math. (2) 85 (1961), 91-96. MR 24 \#A3237.

3. Howard Garland, $p$-adic curvature and the cohomology of discrete subgroups of p-adic groups, Ann. of Math. (2) 97 (1973), 375-423.

4. D. A. Každan, On the connection of the dual space of a group with the structure of its closed subgroups, Funkcional. Anal. i Priložen. 1 (1967), 71-74. (Russian) MR 35 \#288.

5. S. P. Wang, On a theorem of representation of lattices, Proc. Amer. Math. Soc. 23 (1969), 583-587. MR 40 \#1530.

6. 921-937. MR 41 \#3665.

7. - On isolated points in the dual spaces of locally compact groups (to appear).

8. A. Weil, Remarks on the cohomology of groups, Ann. of Math. (2) 80 (1964), 149-157. MR 30 \#199.

9. H. Whitney, Elementary structure of real algebraic varieties, Ann. of Math. (2) 66 (1957), 545-556. MR 20 \#2342.

Department of Mathematics, State University of New York, Stony Brook, NEW YORK 11790

Current address: Department of Mathematics, Purdue University, Lafayette, Indiana 47907 